\title{
Malaria in Nonimmune Travelers: A Synopsis of History, Symptoms, and Treatment in 160 Patients
}

\author{
Tomas Jelinek, H.D. Nothdurft, and T. Löscher
}

Background: With the current increase of international travel to tropical endemic areas, the incidence of malaria being imported into nonendemic countries has increased significantly. Disagreement concerning malaria chemoprophylaxis and inadequate knowledge of malarious areas, morbidity, and pretravel advise has led to confusion among both health professionals as well as travelers. Therefore, this study was conducted to investigate malaria imported into Germany by identifying the high-risk endemic areas, clinical presentations, and chemoprophylactic and therapeutic regimens related to reported cases.

Methods: Between 1990 and 1993, the 160 nonimmune travelers, all German nationals or residents for more than 10 years, presenting to our travel clinic with microscopically confirmed malaria were investigated. For each, the travel history, chemoprophylaxis used during travel, symptoms, pathological diagnosis, and treatment efficacy were analyzed.

Results: Africa (73\%), Asia (21\%), and Central South America (6\%) were the endemic countries visited by our patients, of whom only $3 \%$ used the chemoprophylaxis recommended for their destination. Plasmodium falciparum was the most common pathogen, found in more than half of our patients, and $P$. vivax (29\%), $P$. ovale (6\%), P. malariae (6\%), a mixed infection with $P$.falciparum and $P$ vivax $(3 \%)$ were also detected. All patients presented with fever and headaches, a majority with profuse night sweats, insomnia, arthralgias, and myalgias, and diarrhea and abdominal cramps were experienced in $13 \%$ and $8 \%$, respectively. In falciparum malaria, a recrudescence was observed in all patients who received chloroquine only, whereas quinine, halofantrine, and mefloquine were highly effective. In vivax malaria, a relapse rate of $14 \%$ was noted in the patients treated with the currently recommended regimen of chloroquine and primaquine.

Conclusions: Visitors to endemic countries, especially to Africa, are of significant risk. Given the low compliance rate of chemoprophylaxis, a high percentage of malaria in our patients could have been avoided by an appropriate prophylaxis regimen and optimal pretravel counseling. ( $J$ Travel Med 1:199-202, 1994)

Malaria presents a serious hazard to travelers to endemic areas. As international air travel to tropical destinations becomes more and more popular, an increase in imported cases has been observed in nonendemic countries in recent years. ${ }^{1-3}$ The importance of appropriate drug prophylaxis has been stressed previously. ${ }^{4-7}$ Chemoprophylaxis should be adapted to each traveler's individual characteristics, the travel itinerary, and the drugs used. ${ }^{8}$ The risk of malaria infection varies depending on the destination and the exposure to infected mosquitos. Therefore, a profound knowledge of malarious areas and the frequency of infection acquired by nonimmune travelers is necessary when advice about prophylaxis is given by a physician. There is some disagreement about malaria drug prophylaxis for travelers to endemic regions. ${ }^{9}$ This leads to considerable diversity in the recommended drug regimens used and in confusion among both health professionals and travelers.

Tomas Jelinek, MD, H.D. Nothdurf, MD, and T. Löscher, MD: Department of Infectious Diseases and Tropical Medicine, University of Munich, Munich, Germany.

Reprint requests: T. Jelinek, MD: Department of Infectious Diseases and Tropical Medicine, University of Munich, Leopoldstr. 5, 80802 Munich, Germany
In this study, a total of 160 patients who presented with imported malaria were investigated. The objective of the investigation was to optimize counseling concerning malaria and also to obtain more information about malaria imported to Germany. The intention was to identify areas with high risk for malaria infection for travelers, common clinical presentations, and the use of prophylactic and therapeutic regimens.

\section{Patients and Methods}

Twenty percent of the total patient population of the travel clinic under investigation present with fever. Malaria is diagnosed in approximately $3 \%$ of the cases, with fever providing the guiding symptom. The case histories, symptoms, and treatment of $160 \mathrm{pa}-$ tients with microscopically confirmed malaria, who were treated in our travel clinic during the period from 1990 to 1993 , were investigated. A thorough travel history was stressed as was knowledge of prophylactic antimalarial drugs used during the journey. A close follow up to detect any relapses was also stressed.

\section{Results}

All patients were German nationals or had been residents of Germany for more than 10 years. One 

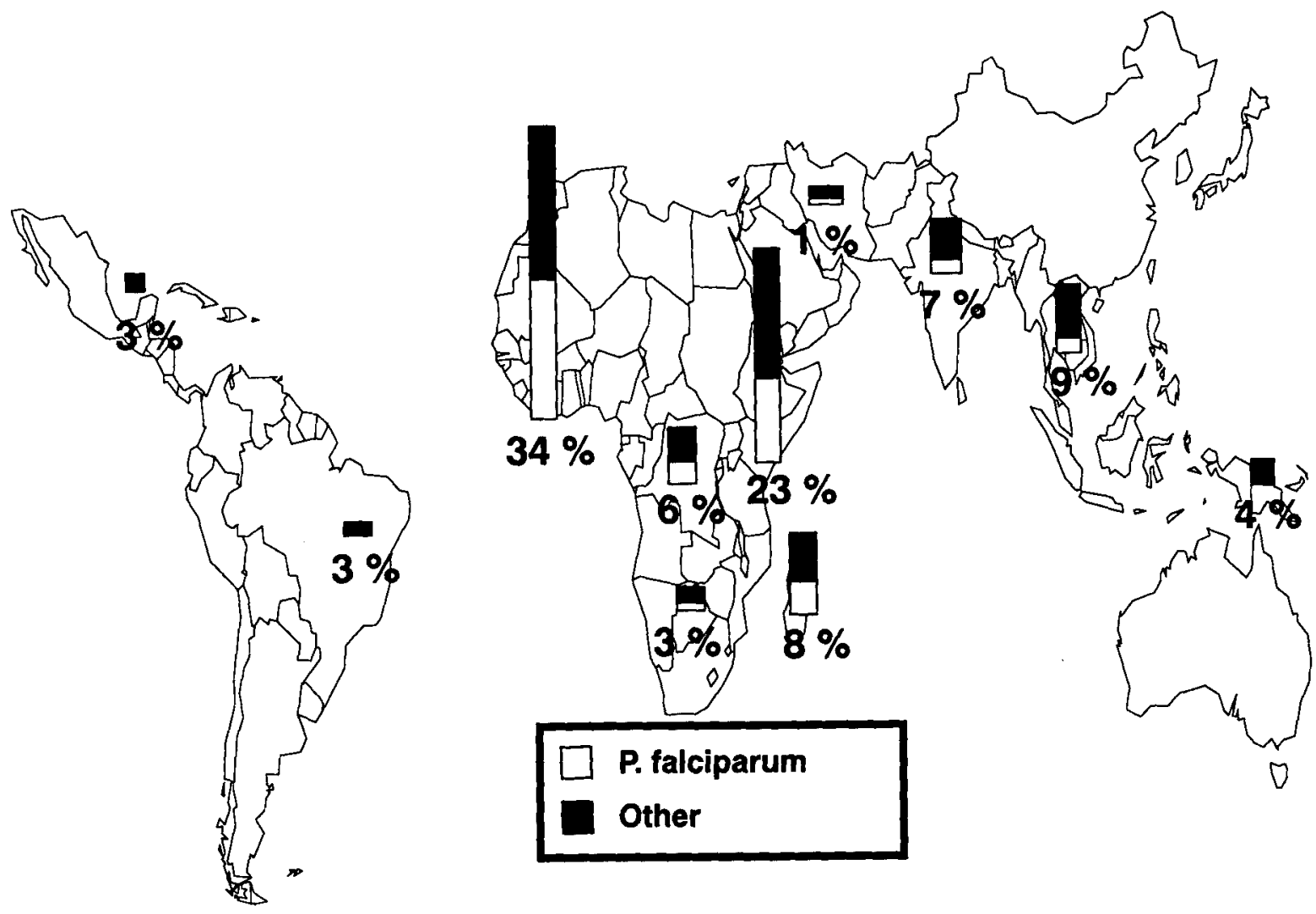

Figure 1 Malaria in nonimmune travelers: geographic distribution in 160 patients.

hundred and sixteen $\{73 \%$ ) of the patients were male and $44(28 \%)$ were female. Ages varied between 15 months and 70 years (mean value 34.5 years, median 32 years). The duration of travel varied from short journeys of 6 days to lengthy stays of up to 19 years (median 28 days). In $88(55 \%)$ of the patients, a diagnosis of falciparum malaria was established, $47(29 \%)$ had vivax malaria, $10(6 \%)$ ovale malaria, $10(6 \%)$ quartan malaria and $5(3 \%)$ a mixed infection of falciparum and vivax malaria. Investigation of the countries where the infection was acquired, revealed an obvious predominance of African destinations, especially for the infection with falciparum malaria (Fig. 1). Thirty-four percent (confidence interval (95\%): 31$6.4 \%$ ) of all malaria cases had previously been to West Africa, $23 \%$ (confidence interval (95\%): $20.5-25.7 \%$ ) to East Africa, $6 \%$ (confidence interval (95\%): 3$8.3 \%$ ) to Central Africa, 3\% (confidence interval (95\%): 0.4-5.8\%) to Southern Africa and further $8 \%$ (confidence interval (95\%): 5.5-10.9\%) to Madagascar. So altogether, Africa contributed $73 \%$ of all malaria patients compared to $21 \%$ (confidence interval (95\%): 18-23.3\%) from Asia and 6\% (confidence interval (95\%): 3-8.3\%) from the Americas. No falciparum malaria was acquired in South America, whereas $50 \%$ of the malaria cases from West Africa were infected with $P$. falciparum.

In $100 \%$ of the patients, symptoms consisted of fever and headache and were usually accompanied by other complaints such as general weakness $(94 \%)$, profuse sweating at night $(91 \%)$, insomnia $(69 \%)$, arthralgia $(59 \%)$, myalgias $(56 \%)$, diarrhea $(13 \%)$, and abdominal cramps (8\%).

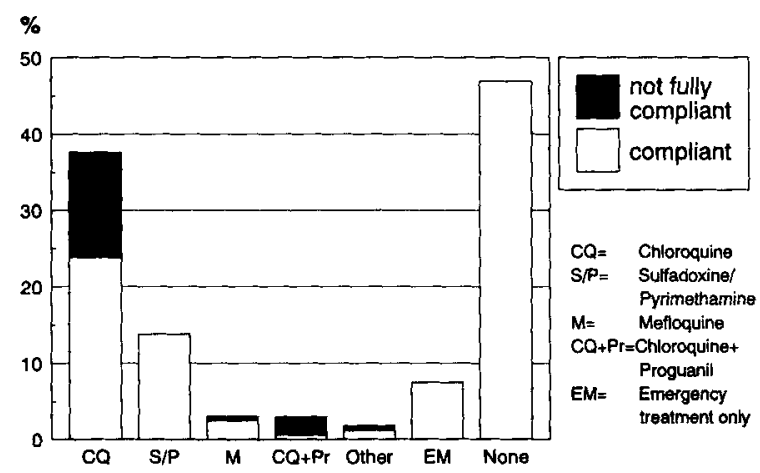

Figure 2 Prophylactic regimen used by 160 travelers with malaria $(\%)$. 
The duration of symptoms before diagnosis was confirmed depended largely on the form of malaria acquired. In falciparum malaria, $81 \%$ of the patients were diagnosed in the first week and the other $19 \%$ within 2 weeks after onset of symptoms. In vivax malaria, diagnosis was established in only $64 \%$ of the patients within 2 weeks. In 5 patients $(11 \%)$, it took between 7 and 61 months, with returning febrile attacks, before the diagnosis of vivax malaria was confirmed. Patients with ovale or quartan malaria were, in $80 \%$ of the cases, diagnosed within 3 weeks, but it took between 2 and 6 months in 2 patients (20\%) before diagnosis was established.

The investigation of the type of chemoprophylaxis used revealed a high percentage of incomplete or insufficient regimens. Only 3\% used the chemoprophylaxis appropriate for the drug resistance situation of malaria parasites at their destination. Forty-seven percent of the patients used no prophylactic regimen at all, and $8 \%$ carried a standby medication only. Chloroquine was used by 60 (38\%) of the 160 investigated patients as the prophylactic drug of choice. However, $37 \%$ of patients using chloroquine disrupted the intake regimen at some point of their journey (Fig. 2).

Twenty six percent of patients had been treated prior to referral to our clinic. This had led to 17 different drugs and drug combinations being used for therapy. All patients with falciparum malaria, who received chloroquine only, showed a recrudescence within 2-8 days (mean 3.5 days), whereas treatment with quinine presented with a curative efficacy of $100 \%$. Halofantrine and mefloquine were effective in $94 \%$ and $98 \%$ of cases, respectively. A high relapse rate was common in patients infected with $P$. vivax, who were treated with combinations without primaquine $(100 \%$ in patients treated with halofantrine, mefloquine, or tetracycline; $75 \%$ in patients treated with chloroquine). These patients presented with relapses within 5 days and 4 months (mean 21 days). However, even in the $14 \%$ of patients treated with the usually recommended combination of chloroquine and primaquine, ${ }^{4}$ relapses did occur within 1 and 5 months (mean 75 days).

\section{Discussion}

Although only $9 \%$ of our total patient population had been to West Africa prior to referral, $34 \%$ of the patients with malaria had acquired the infection there. Similar results were obtained for East Africa and Madagascar: 10 percent of our total patient population traveled to these areas. Thirty-one percent of the patients with malaria had been previously to East
Africa or Madagascar. The risk of acquiring malaria in South America and Southeast Asia seems lower. The figures here were $3 \%$ of the malaria-infected patients from a total of $9 \%$ of patients who traveled there. Twenty percent of all patients traveled to Southeast Asia, with only $9 \%$ becoming infected. Obviously, the risk of infection is highest in tropical Africa. These findings are comparable to previous investigations: in these the risk of malaria infection per traveler was calculated at 1:530 for tropical Africa, 1:1920 for India, 1:4170 for Southeast Asia, 1:9090 for Central America and 1:25000 for South America. ${ }^{1,3,6,12-14}$ Only $3 \%$ of all patients took drugs or drug combinations appropriate for the drug resistance situation of malaria parasites at the respective destination. ${ }^{4}$ Probably, a high percentage of the malaria cases discussed here could have been avoided by an appropriate malaria prophylaxis regimen.

The duration of symptoms might be viewed as a measure of the diagnostic efficacy of the health professionals involved. All patients presented with clinical symptoms typical of malaria, mainly attacks of fever and headache. The diagnosis of falciparum malaria within the first week after onset of complaints in $81 \%$ of the patients is certainly due to the usually severe symptoms in the presentation of this disease. The delay of up to 61 months in the diagnosis of vivax, ovale, and quartan malaria demonstrates the importance of the consideration of malaria in the differential diagnosis of fever, even if a journey to an endemic area took place several weeks ago.

The failure rate in the different therapeutic regimens used is notable. Although the sample size is rather small, in some of the treatment groups, one clear result is the demonstration of the inadequacy of chloroquine for the treatment of falciparum malaria acquired in Africa. Mainly for financial reasons, chloroquine is still the first line drug of choice in many developing countries. ${ }^{15}$ In semiimmune patients, chloroquine ameliorates the course of disease and might even result in clinical cure. In nonimmune patients, however, the aim of malaria treatment must be to complete the eradication of the parasites, since even low parasitemias evoke clinical disease. All patients in this investigation treated with chloroquine only had already received therapy in Africa. Therefore, it is advisable to carry a potent antimalarial drug for emergency treatment when traveling to malarious countries. Primaquine is highly effective in the prevention of relapses in vivax malaria as is demonstrated by the results in this study population. However, the relapse rate of $14.1 \%$ in patients treated with chloroquine and primaquine shows that relapses have to be expected to a certain extent. 


\section{References}

1. Philips-Howard PA, Radalowicz A, Mitchell J, Bradley DJ. Risk of malaria in British residents returning from malarious areas. BMJ 1990; 300:499-503.

2. Kollaritsch H, Wiedermann G. Compliance of Austrian tourists with prophylactic measures. Eur J Epidemiol 1992; 8:243-251.

3. Froude JR, Weiss LM, Tanowitz HB, Wittner M. Imported malaria in the Bronx: review of 51 cases recorded from 1986 to 1991. Clin Infect Dis 1992; 15:774-780.

4. World Health Organization. International travel and health. Geneva: World Health Organization, 1994.

5. Steffen R, Heusser R, Mächler R, et al. Malaria chemoprophylaxis among European tourists in tropical Africa: use, adverse reactions, and efficacy. Bull World Health Organ 1990; 68:313-322.

6. Steffen R, Fuchs E, Schildknecht J, et al. Mefloquine compared with other malaria chemoprophylactic regimens in tourists visiting East Africa. Lancet 1993; 341:1299-1300.

7. Lobel HO, Bernard KW, Williams SL et al. Effectiveness and tolerance of long-term malaria prophylaxis with mefloquine. JAMA 1991; 255:361-364.
8. Baudon D. Malaria and travelers-practical aspects. Trop Med Parasitol 1993; 44:246-249.

9. Lobel HO, Keystone JS. Confusion on malaria chemoprophylaxis (Letter). Lancet 1994; 343:183.

10. Nathwani D, Badial R, Khaund RR, et al. Malaria in Aberdeen: an audit of 110 patients admitted between 1980-1991. Scott Med J 1992; 37:106-110.

11. Boreham RE, Relf WA. Imported malaria in Australia. Med J Aust 1991; 155:754-757.

12. Lobel HO, Philipps-Howard PA, Brandling-Bennett AD, et al. Malaria incidence and prevention among European and North American travellers to Kenya. Bull World Health Organ 1990; 68:209-215.

13. Steffen R, Behrens RH. Travellers' malaria. Parasitol Today 1992; 8:61-66.

14. Dieckmann SB, Weinke T, Zastrow KD, Schönberg I. Recent epidemiological trends in imported malaria to Germany-an evaluation of the period 1980-1991. Third Conference on International Travel Medicine, Paris, France 1993. (Abstract No. 85)

15. de Glanville $\mathrm{H}$. Bilingual dialogue on malaria. Lancet 1994;343:593.

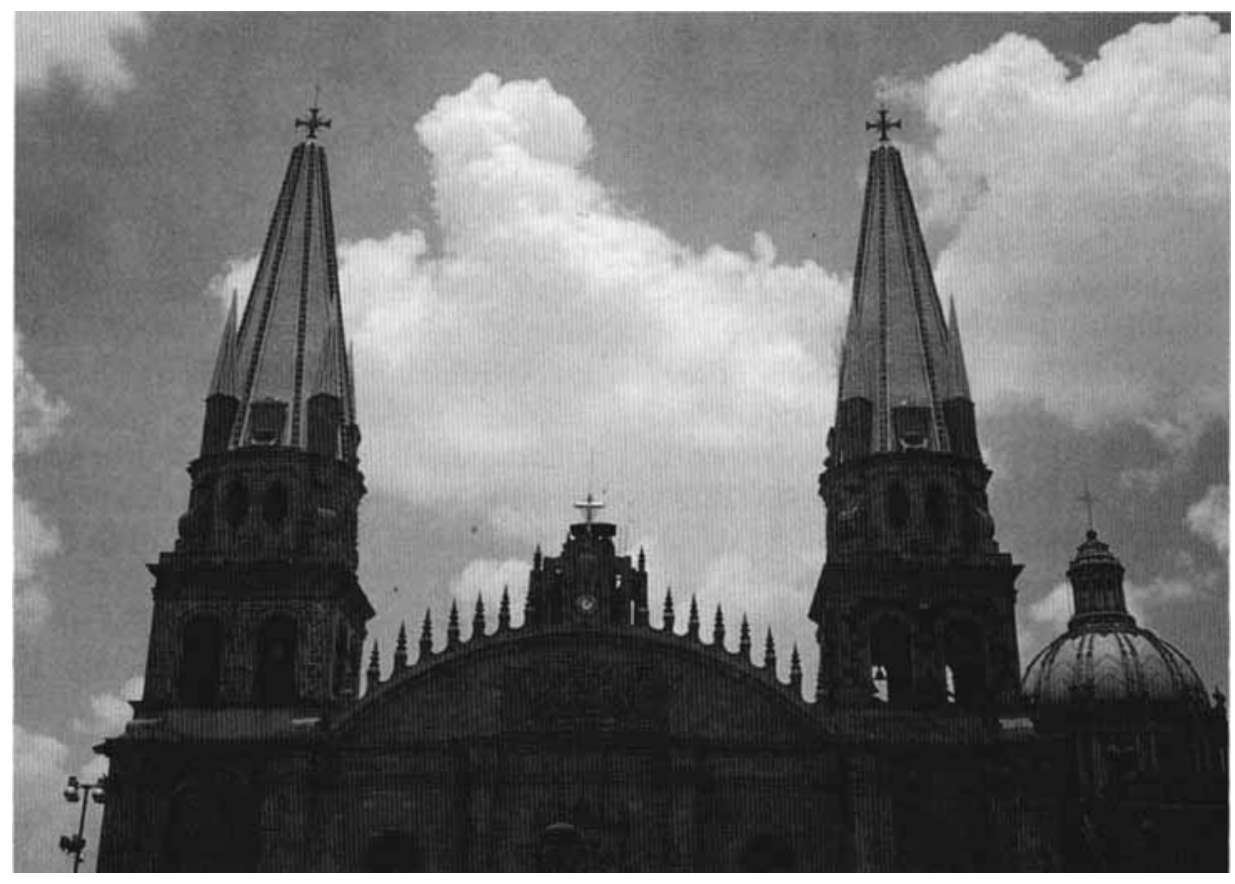

The Cathedral, Guadalajara, Mexico (Submitted by Charles D. Ericsson, MD) 